\title{
A Study on Oil Pipeline Risk Assessment Technique Based on Fuzzy Analytic Hierarchy Process
}

\author{
Hang Dong ${ }^{*}$, Lixin Wei and Qiannan Wang \\ Key Laboratory of Enhance Oil and Gas Recovery of Educational Ministry, Northeast Petroleum University, Daqing, \\ P.R. China
}

\begin{abstract}
Risk assessment of oil pipeline is the core content in the pipeline integrity management. Through risk assessment of oil pipelines, we could know the comprehensive operating condition of the pipelines, identify the dangerous factors of the pipeline and find out the risk section of the pipeline, so as to work out the risk mitigation measures and provide a theoretical basis for integrity assessment of the pipeline. However, the commonly used Kent risk assessment method does not have a very reasonable distribution of the failure indicator values. Therefore, this paper establishes a failure indicator value adjustment model for oil pipeline risk assessment based on the fuzzy analytic hierarchy process. Risk assessment of Qingha Oil Pipeline has been carried out based on the foundation data and real condition of the pipeline, and the established model was used for weighting adjustment of the various failure risk factors of the pipeline. Thus, a risk assessment model which is more suitable for Qingha Oil Pipeline was obtained. This laid a very solid foundation for proposal of suggestions on mitigation of risk of damage by the third party and the implementation of protective measures of the pipeline.
\end{abstract}

Keywords: Damage by the third party, Fuzzy analytic hierarchy process, Risk assessment, Weighed adjustment, Hazard factor.

\section{INTRODUCTION}

At present, the pipeline management models used in many oil and gas transmission pipelines are a kind of passive management models, which take remedial measures only after the problem occurs, and thus makes the safety management quite passive. In recent years, the pipeline integrity management model has been adopted to change this awkward situation. The original passive management method has been changed into an active one so that it advocates identification and assessment of risk factors around the pipeline that are being changed, formulation of related risk control measures and constant mitigation of risk factors doing harm to the pipeline. This way, the risk level could be controlled within a reasonable and acceptable scope. Pipeline risk assessment is the core content of the integrity management of the entire pipeline. Through pipeline risk assessment, various dangerous factors affecting the pipeline and key points of pipeline management could be identified, so as to facilitate control and prevention of risks and guarantee safe operation of the pipeline [1]. The commonly used semi-quantitative assessment method, Kent method divides the failure indicators into 4 groups, namely, damage by the third party, corrosion, design and misoperation, each having 100 scores. This risk indicator score distribution method renders Kent risk assessment method too universal to be pertinent, especially for the pipeline which is dominated by one failure factor. The calculated risk value can not satisfactorily reflect the risk level of the pipeline. Therefore, it is necessary to carry out

*Address correspondence to this author at the Developing Road 199, Hightech Development Zone, Daqing, 163318, P.R. China

Tel: 8613674598630; E-mail: dh.123@163.com weighting adjustment of the risk factors to ensure the method suits the real condition of the pipeline better.

\section{RISK ASSESSMENT MODEL}

\subsection{Foundation Data Collection}

This includes the basic parameters of the pipeline, basic parameters of the oils transmitted through the pipeline, and route and basic tendency of the pipeline (including the location where the pipeline crosses a road, railway or a river), condition of the pipeline yard and the valve chamber, internal inspection data, number of times of historical leakage and conditions, corrosion condition (including the cathodic protection condition, soil corrosion condition and anti-corrosion layers), basic data on damage by the third party, geological disaster, misoperation and design data (including the welding joint defects).

\subsection{Hazard Factor Identification}

This includes four groups of risk indicators, namely, damage by the third party, corrosion, design and misoperation, each having 100 scores.

\subsection{Establishment of Risk Assessment Model}

Relative risk value $=($ exponential sum $) /($ influence coefficient of leakage $)=[($ indicators of damage by the third party $)+($ corrosion indicator $)+($ design indicator $)+($ design indicator $)+($ misoperation indicator) $] /$ influence coefficient of leakage

Influence coefficient of leakage $=$ (product danger) $/$ (diffusion coefficient) 
Product danger $=$ sudden danger $\left(N_{f}+N_{r}+N_{h}\right)+$ longterm danger

Diffusion coefficient $=$ (leakage score $) /($ population score)

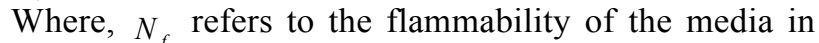
the pipeline; $N_{r}$ refers to the activity of the media in the pipeline; $N_{h}$ refers to the toxicity of the media in the pipeline.

\subsection{Pipeline Section Risk Calculation}

The risk calculation formula of each pipeline section is as follows:

$$
R=\sum_{k=1}^{5}\left(L_{k} / C_{k}\right)
$$

Where, $R-$ relative risk value; $L-$ failure possibility score; $C$ - failure consequence; $k$ failure cause (corrosion, damage by the third party, manufacturing and construction defects, misoperation, or geological disaster). Specifically, $C k$ represents the consequence scores under each failure mode, and is related to the leakage amount, acceptor, diffusion coefficient, and product danger; the lower the failure possibility score is, the more danger there will be. The higher the consequence score is, the more serious the consequence will be; a smaller risk value indicates a greater risk.

\subsection{Overall Relative Risk Value of the Pipeline} line:

Computational formula of the overall risk of the pipe-

$$
R=\sum_{j=1}^{J}\left(R_{j} \times l_{j}\right) / l
$$

Where, $R$ - refers to the relative risk value of the pipeline; $j$-number of pipe sections; $j=1,2, \cdots, J ; R_{j}-$ relative risk value of section $j ; l_{j}$-length of section $j ; l$ - total length of the sections

\section{WEIGHT ADJUSTMENT METHOD}

The pipeline risk assessment scholars usually use two kinds of thoughts to determine the weight of the risk factor scores, which are respectively the analytic hierarchy process used in system engineering and gray relative analysis method in the grey system theory. Those two types of thought both advocate determination of weight through rigid mathematical method to reduce the impact of subjective factors [2]. Specifically, the fuzzy analytic hierarchy process is more suitable for the hierarchical structure characteristics of oil pipeline risk factors and compared with the gray relative analysis method, the former could obtain the scores after risk factor weight adjustment, rather than the value-based order of the factor weights obtained through non-gray relative analysis method, in an easier manner. Therefore, in this paper, the fuzzy analytic hierarchy process was used to work out the adjustment of risk assessment factor weight for oil pipelines.

The Analytic Hierarchy Process is called AHP for short, and was proposed by a well-known American opera- tional research expert from University of Pittsburgh named T.L.Saaty in the middle of the 70s [3, 4]. The model and steps of using AHP to calculate the pipeline risk factor weight are as follows:

(1) Establishment of a hierarchy analytical structure of the risk factors $[5,6]$

The risk factor hierarchies mainly include the target layer (A), the criteria layer (B) and the factor layer (C and D).

\section{(2) Building of the fuzzy risk judgment matrix}

Assume the Element B in the previous layer is related to Elements $c_{1}, c_{2}, \cdots, c_{n}$ of the next later, then the following fuzzy risk judgment matrix could be built up:

$$
\left[\begin{array}{cccc}
a_{11} & a_{12} & \ldots & a_{1 n} \\
a_{21} & a_{22} & \ldots & a_{2 n} \\
\ldots & \ldots & \ldots & \ldots \\
a_{n 1} & a_{n 2} & \ldots & a_{n n}
\end{array}\right]
$$

Where, $a_{i j}=\left(l_{i j}, m_{i j}, u_{i j}\right)$ is a triangular fuzzy number, and $l_{i j}, m_{i j}, u_{i j}$ are respectively the most pessimistic estimate, the most possible estimate and the most optimistic estimate of the importance of relative factor $c_{i}$ and relative risk factor $c_{j}$ proposed by the expert after comparison between risk factors $c_{i}$ and $c_{j}$ with respect to the risk factor $B$.

\section{(3) Weight calculation of fuzzy judgment matrix}

In a hierarchical structure, the weight refers to the relative intensity of the impact of different elements on a lower layer onto the elements on a higher layer or the general objective. By calculating the triangular fuzzy number complementary judgment matrix row SNORM, the weight vector of its triangular fuzzy number could be obtained and then the weight vector of its triangular fuzzy number could also be obtained.

$$
w=\left(w_{1}, w_{2}, w_{3}, w_{4}, w_{5}\right)^{T}
$$

Make pair-wise comparison between the triangular fuzzy numbers $w_{i}$, and calculate the possibility degree $p_{i j}\left(w_{i} \geq w_{j}\right)$ and establish the possibility degree matrix $P=\left(p_{i j}\right)_{n \times n}$.

Then the solution to the ordering vector of the possibility degree matrix will be the order of the triangular fuzzy numbers.

$$
\begin{gathered}
\omega_{i}=\frac{2}{n^{2}} \sum_{j=1}^{n} p_{i j} \quad i \in N \\
\omega=\left(\omega_{1}, \omega_{2}, \omega_{3}, \ldots, \omega_{n}\right)^{T}
\end{gathered}
$$

Where,

$$
\omega_{1}, \omega_{2}, \omega_{3}, \omega_{4}, \omega_{5}
$$

are respectively the weight ratio of the impact of each risk factor on the pipeline failure. The score distribution formula of each risk factor is as follows: 


$$
X_{i}=100 k \omega_{i} i=1,2,3, \ldots, k
$$

(4) Adjustment of failure possibility scores in the risk assessment

For pipelines with a risk assessment result, its risk factor scores could be adjusted. If the original risk assessment calculation result is $x_{i}$ ( $i$ refers to the risk factor, $i=1,2, \ldots, k$ ) and the adjusted risk factor result is $x_{i}$, then the calculation formula should be as follows:

$$
\begin{gathered}
x_{i}^{\prime}=k \omega_{i} x_{i} \\
i=1,2,3, \ldots, k
\end{gathered}
$$

\section{BASIC INFORMATION OF THE PIPELINE}

By collecting the historical data on pipeline leakage according to the existing data on the pipeline, it was found that the number of times of leakage of the pipeline during 2005 and 2012 was 91 . Specifically, the pipeline leakage caused by damage by the third party accounts for $53 \%$ of the total, $42 \%$ accounts for corrosion, while manufacturing and construction defects account for 5\%. The main reasons that resulted in pipeline failure mainly include damage by the third party, corrosion and manufacturing and construction factors. The number of leakages caused by damage by the third party accounts for more than $50 \%$ of the total, which indicates that Qingha Oil Pipeline was subject to serious damage by the third party. Therefore, for distribution of risk factor scores, the score of this risk factor should differ from that of others so as to ensure a more scientific and accurate result. As such, the fuzzy analytic hierarchy process was used to adjust the risk factor score weight for Qingha Oil Pipeline.

\section{PIPELINE RISK ASSESSMENT BASED ON AN IMPROVED METHOD}

\subsection{Risk Calculation Result Before Weight Adjustment}

Through risk assessment calculation for Qingha Oil Pipeline, the scores of each item of failure possibility are given below:

Table 1. Failure possibility indicator scores.

\begin{tabular}{|c|c|}
\hline Failure Possibility Indicator & Scores \\
\hline \hline Damage by the third party & $42 \sim 84$ \\
\hline Corrosion & $57 \sim 73$ \\
\hline Misoperation & $82 \sim 92$ \\
\hline $\begin{array}{c}\text { Manufacturing and construction } \\
\text { defect }\end{array}$ & $61 \sim 83$ \\
\hline Geological disaster & $75 \sim 98$ \\
\hline
\end{tabular}

As shown in the Table 1, the scores of failure possibility indicators of damage by the third party and corrosion factors are low and are main reasons for pipeline failure. This coincides with the characteristics of the leakage history of the pipeline that the damage by the third party and corrosion are frequent.

\subsection{Risk Calculation Based on Weight Adjustment Method}

To get a more accurate fuzzy comparison result, the historical failure record of Qingha Oil Pipeline is reviewed and the risk probability was calculated using the method proposed by United States Nuclear Regulatory Commission:

$$
\begin{aligned}
& r=M /(N L) \\
& P_{f}(t)=1-e^{-r t}
\end{aligned}
$$

Where, $L-$ length of the pipeline, $\mathrm{km} ; N-$ time section, $a ; M-$ number of times of failure occurring during the $N$ section, times; $r$ - failure frequency, time $/ L . a$ ); $t$ — time of use, $a ; P_{f}(t)$ — risk probability, \%;

According to the number of times of historical failures of Qingha Oil Pipeline during 7 years from 2005 to 2012, the frequency and risk probability of pipeline failure caused by different risk factors were obtained and shown in Table 2.

Table 2. Risk probability of pipeline failure caused by different risk factors.

\begin{tabular}{|c|c|c|c|}
\hline Risk Factors & $\begin{array}{c}\text { Total Failures } \\
\text { During the 7 } \\
\text { Years (times) }\end{array}$ & $\begin{array}{c}\text { Failure } \\
\text { Frequency } \\
\text { (time/L.a) }\end{array}$ & $\begin{array}{c}\text { Risk } \\
\text { Probability } \\
\text { (\%) }\end{array}$ \\
\hline \hline $\begin{array}{c}\text { Damage by the third } \\
\text { party } \mathrm{B}_{1}\end{array}$ & 48 & 0.0374 & 3.67 \\
\hline Corrosion $\mathrm{B}_{2}$ & 38 & 0.0296 & 2.92 \\
\hline $\begin{array}{c}\text { Misoperation } \mathrm{B}_{3} \\
\text { Manufacturing and } \\
\text { construction defect } \\
\mathrm{B}_{4}\end{array}$ & 0 & 0 & 0 \\
\hline $\begin{array}{c}\text { Geological disaster } \\
\mathrm{B}_{5}\end{array}$ & 0 & 0.0031 & 0.31 \\
\hline
\end{tabular}

According to the risk probability of each risk factor and the $0.1 \sim 0.9$ scaling method obtained through calculation, the following fussy judgment matrix could be obtained:

$\left[\begin{array}{lllll}b_{11} & b_{12} & b_{13} & b_{14} & b_{15} \\ b_{21} & b_{22} & b_{23} & b_{24} & b_{25} \\ b_{31} & b_{32} & b_{33} & b_{34} & b_{35} \\ b_{41} & b_{42} & b_{43} & b_{44} & b_{45} \\ b_{51} & b_{52} & b_{53} & b_{54} & b_{55}\end{array}\right]$
$=\left[\begin{array}{llllll}(0.5,0.5,0.5) & (0.6,0.6,0.7) & (0.7,0.8,0.9) & (0.7,0.7,0.8) & (0.8,0.8,0.9) \\ (0.3,0.4,0.4) & (0.5,0.5,0.5) & (0.8,0.8,0.9) & (0.7,0.7,0.8) & (0.7,0.8,0.8) \\ (0.1,0.2,0.3) & (0.1,0.2,0.2) & (0.5,0.5,0.5) & (0.3,0.4,0.4) & (0.4,0.4,0.6) \\ (0.2,0.3,0.3) & (0.2,0.3,0.3) & (0.6,0.6,0.7) & (0.5,0.5,0.5) & (0.6,0.6,0.8) \\ (0.1,0.2,0.2) & (0.2,0.2,0.3) & (0.4,0.6,0.6) & (0.2,0.4,0.4) & (0.5,0.5,0.5)\end{array}\right]$

Through calculation of fuzzy judgment matrix, the relative weight vector of the fuzzy risk factors could be obtained:

$$
\begin{aligned}
& w=\left(w_{1}, w_{2}, w_{3}, w_{4}, w_{5}\right)^{T} \\
& =\left(\begin{array}{l}
(0.295,0.272,0.275),(0.268,0.256,0.246), \\
(0.125,0.136,0.145),(0.188,0.184,0.188),(0.125,0.152,0.145)
\end{array}\right)^{T}
\end{aligned}
$$


The possibility degree matrix has been established as follows according to the possibility degree calculation formula:

$$
P=\left[\begin{array}{ccccc}
0.5 & 1 & 1 & 1 & 1 \\
0 & 0.5 & 1 & 1 & 1 \\
0 & 0 & 0.5 & 0 & 0.145 \\
0 & 0 & 1 & 0.5 & 1 \\
0 & 0 & 0.855 & 0 & 0.5
\end{array}\right]
$$

The following sort algorithm is used to sort the risk factors:

$$
\omega_{i}=\frac{2}{n^{2}} \sum_{j=1}^{n} p_{i j}(4-4)
$$

The relative weight vector $\boldsymbol{\omega}$ of the relative risk factors was obtained:

$$
\begin{aligned}
& \omega=\left(\begin{array}{lll}
\omega_{1}, \omega_{2}, \omega_{3}, \omega_{4}, \omega_{5} & )^{T} \\
=(0.36,0.28,0.0516,0.2,0.1084
\end{array}\right)^{T}
\end{aligned}
$$

This means that the weight ratios of damage by third party, corrosion, misoperation, manufacturing and construction defects and geological disaster for the pipeline failure are respectively $0.36,0.28,0.0516,0.2$ and 0.1084 , and the damage by third party $>$ corrosion $>$ misoperation $>$ manufacturing and construction defects $>$ geological disaster.

If the total scores are 500, then the distribution of scores of each item is shown in Table $\mathbf{3}$ :

Table 3. Table of distribution of scores of each risk factor of Qingha Oil Pipeline.

\begin{tabular}{|c|c|c|}
\hline $\begin{array}{c}\text { Damage by the Third Party } \\
\mathbf{B}_{\mathbf{1}}\end{array}$ & Weight Ratio & Scores \\
\hline \hline Corrosion $\mathrm{B}_{2}$ & 0.36 & 180 \\
\hline $\begin{array}{c}\text { Misoperation } \mathrm{B}_{3} \\
\begin{array}{c}\text { Manufacturing and construc- } \\
\text { tion defect } \mathrm{B}_{4}\end{array}\end{array}$ & 0.28 & 140 \\
\hline Geological disaster $\mathrm{B}_{5}$ & 0.2 & 25.8 \\
\hline Damage by the third party $\mathrm{B}_{1}$ & 0.1084 & 100 \\
\hline Total & 1 & 54.2 \\
\hline
\end{tabular}

The scores of the failure possibility calculation result of the risk assessment were adjusted. The comparison between the failure possibility scores before and after adjustment is given in Figs. (1) to (5):

As shown in the above figures, after adjustment of the score weight of each risk factor of pipeline using the fuzzy analytic hierarchy process, the scores of failure possibility due to damage of third party and corrosion raised sharply after the adjustment. This indicates that the impact of the two risk factors pose a greater impact on the pipeline failure. Among the risk factors, the scores of failure possibility due to manufacturing and construction defect before and after the adjustment are the same. This indicates that the impact of the risk factor on the pipeline failure remains the same. Meanwhile, the sores of failure possibility due to misoperation and geological disaster dropped moderately after adjustment. This indicates that the impact of the two factors on the pipeline failure is subtle. The change of scores related to each risk factor after adjustment coincides with the characteristics of the leakage history of the pipeline that the damage by the third party and corrosion are frequent.

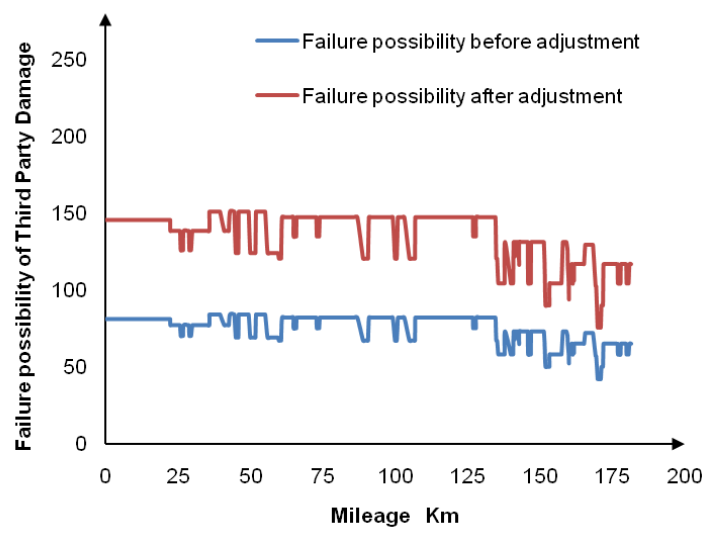

Fig. (1). Comparison between scores before and after adjustment of damage by third party factor.

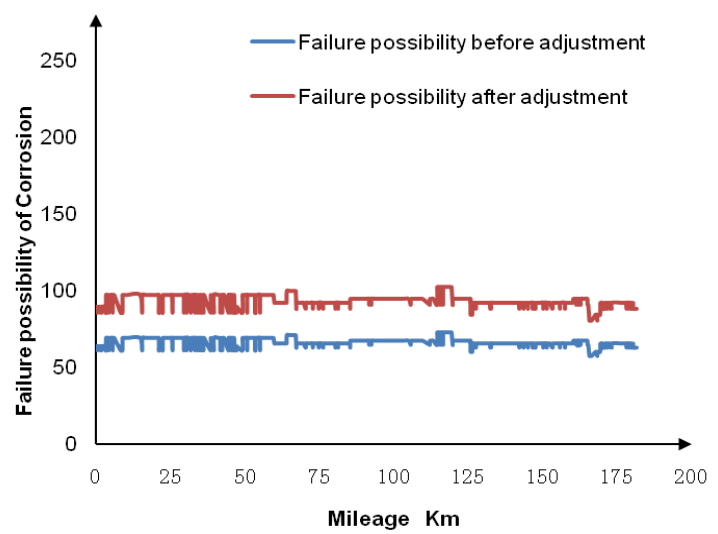

Fig. (2). Comparison between scores before and after adjustment of corrosion factor.

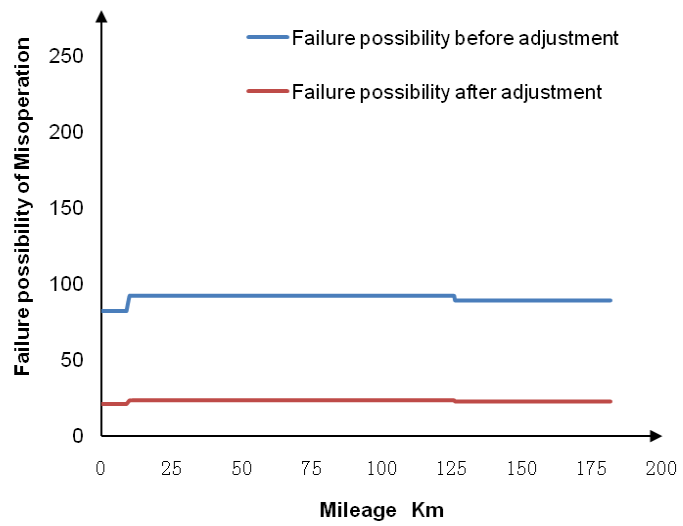

Fig. (3). Comparison between scores before and after adjustment of misoperation factor. 


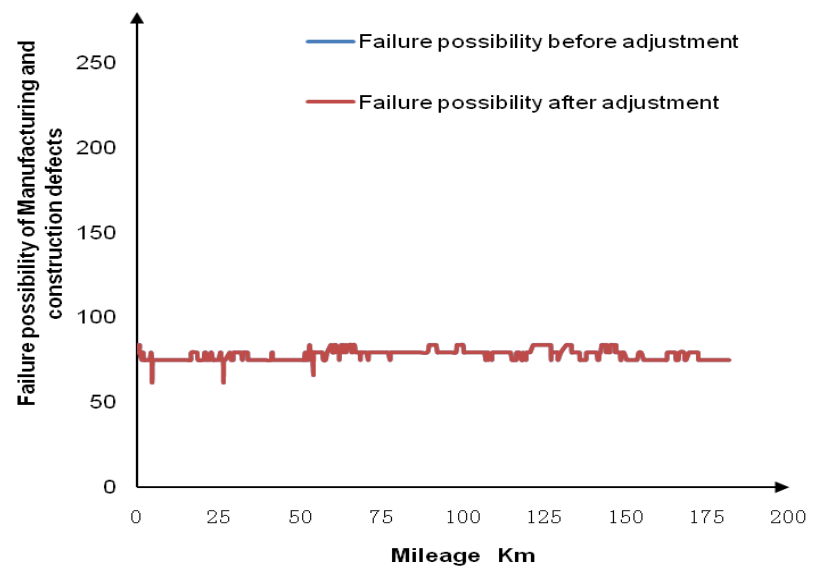

Fig. (4). Comparison between scores before and after adjustment of manufacturing and construction defect factor.

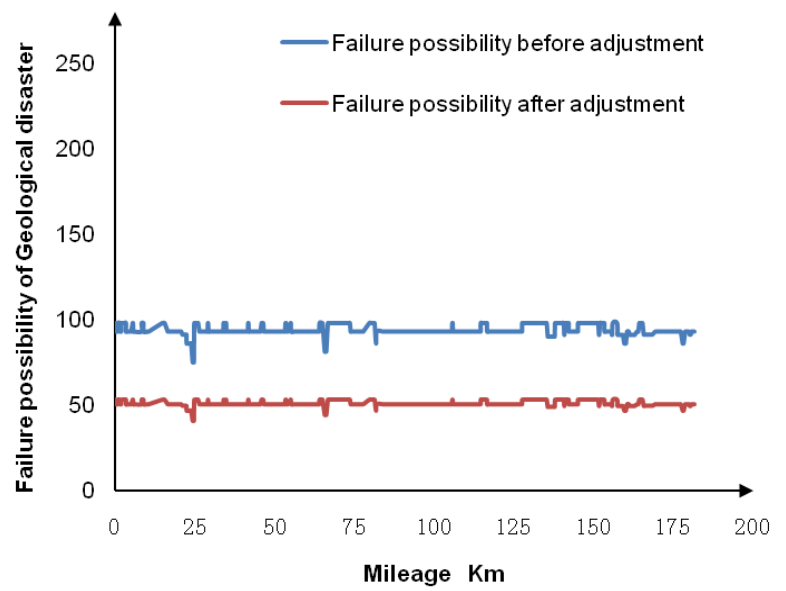

Fig. (5). Comparison between scores before and after adjustment of geological disaster factor.

In Fig. (1), the scores after adjustment of risk factor of damage by third party range from 40 to 120 , and get even greater in the later section of the pipeline, obviously greater than the scores in the front section and middle section of the pipeline. This indicates that the possibility of damage by third party on the later section of Qingha Oil Pipeline is higher than that on the front and middle sections. Therefore, the damage by third party identification and pipeline protec- tion measures should be reinforced in regions with high scores.

In Fig. (2), the scores after adjustment of risk factor of corrosion are around 60. This indicates that the entire pipeline is subject to severe corrosion. Comprehensive repairing plan should be made and implemented for the corroded pipeline to protect the pipeline against being further damaged by corrosion.

\section{CONCLUSION}

This paper established the risk assessment failure indicator scores and risk value adjustment model based on the analytic hierarchy process and the theoretical study results of oil pipeline risk assessment. Risk assessment was conducted on Qingha Oil Pipeline according to its basic data and the real condition of the pipeline. The weight adjustment model was used to adjust the failure indicator scores to obtain a risk assessment model for risk assessment that fits Qingha Oil Pipeline better.

\section{CONFLICT OF INTEREST}

The authors confirm that this article content has no conflict of interest.

\section{ACKNOWLEDGEMENTS}

Declared none.

\section{REFERENCES}

[1] PetroChina Pipeline Company, Integrity Management Techniques for Oil Pipelines, Petroleum Industry Press: Beijing, 2010

[2] Z. Xue, "Combustion and Explosion Theory", 2011.

[3] Y. Shurong, M. Xin, Determination of Pipeline Risk Factor Weight Based on Analytic Hierarchy Process, Natural Gas Industry, 2005.

[4] X. Shubai, Practical Decision-making Method - Principle of Analytic Hierarchy Process, 1988.

[5] C. Liangjun, S. Kechun, Y. Anlin, "Analysis of gas pipeline risk factor weight based on fuzzy analytic hierarchy process", Guangzhou Chemicals vol. 37, no. 8, pp. 12-14, 2009.

[6] C. Liqiong, Zhangpeng, M. Jianlin, "A discussion on fuzzy synthetic evaluation method for oil and gas pipeline risks", Natural Gas Industry, vol. 23, no. 2, pp. 117-119, 2003.

\author{
Received: September 16, 2014 \\ Revised: December 23, 2014 \\ Accepted: December 31, 2014 \\ (C) Dong et al.; Licensee Bentham Open.
}

This is an open access article licensed under the terms of the Creative Commons Attribution Non-Commercial License (http://creativecommons.org/licenses/by-nc/4.0/) which permits unrestricted, non-commercial use, distribution and reproduction in any medium, provided the work is properly cited. 